\title{
PER UNA STORIA DELLA 'LEPIDITAS': IL CASO PERIPLECTOMENO NEL MILES GLORIOSUS E LE SUE RISONANZE DA TERENZIO A APULEIO
}

\author{
Laura Aresi \\ Università degli Studi di Firenze \\ laura.aresi@unifi.it
}

L'articolo si propone di investigare l'uso dell'aggettivo lepidus nel Miles gloriosus, a partire dalla figura del senex lepidus Periplectomeno e della sua aristia in 3.1. L'indagine farà emergere due accezioni del termine: da una parte, lepidus è la parola chiave per indicare il meccanismo del comico su cui si regge la commedia; dall'altra, esso definisce uno stile di vita -e, implicitamente, un modello educativofondato sulla 'lepiditas'. A partire da Plauto, tale modello verrà ripreso attivamente in età repubblicana, per poi venire meno, invece, in età imperiale, dove lepidus va incontro ad un impoverimento semantico e viene usato solo come marcatore di un genere comico minore e disimpegnato.

\section{The 'Lepiditas' and its history: Periplectomenus in the Miles gloriosus AND his influence from Terence to Apuleius}

The article aims to investigate the use of the adjective lepidus in the Miles gloriosus, beginning with the analysis of the senex lepidus Periplectomenus and his aristia in 3.1. Two meanings of the word will be pointed out: on the one hand, lepidus ist the key-word that underlines the way by which the comic effect is reached in the play; on the other, it represents a way of life -and, indirectly, a method of educationthat is based on the 'lepiditas'. After Plautus, this model will be actively reused in the republican age. In the imperial age, instead, the functions and meanings of lepidus will be strictly reduced and the word will be employed just as marker to introduce funny tales. 
T l dibattito sulla 'plautinità' dell'aristia di Periplectomeno nel Miles gloriosus ha impegnato generazioni di critici e filologi, e ormai pare essersi esaurito ${ }^{1}$. I contributi più recenti dichiarano di non voler prendere parte al faticoso riconoscimento del "plautino in Plauto", rifiutandosi di setacciare il testo alla ricerca del o dei modelli greci di cui l'autore si servì, e preferendo considerare l'opera nella sua unità ${ }^{2}$. Pur non ignorando o negando il problema delle vistose incongruenze che la commedia indubbiamente presenta, anche l'analisi qui proposta si inserisce in questa linea di pensiero: se è ragionevole supporre la compresenza di almeno due modelli alla base del Miles gloriosus ${ }^{3}$, come già Friedrich Leo ed Eduard Fränkel hanno mostrato ormai un secolo $\mathrm{fa}^{4}$, pare difficile riuscire a stabilire a quale dei due appartenga l'aristia di Periplectomeno, o ipotizzare, in alternativa, che questa sia una scena di totale invenzione di Plauto. Quello che è certo -e che è stato già ampiamente sottolineato da più fronti- è che il discorso del senex occupa una posizione centrale nel dramma e rappresenta il trait-d'union tra la beffa a Sceledro e quella al miles, costituendo una pausa importante in mezzo al ritmo incalzante degli eventi. Questa semplice constatazione basta a dare ragione a quanti ${ }^{5}$ sono partiti proprio dalla scena 3.1 per avvalorare la tesi dell'unitarietà del Miles, a prescindere dal numero dei modelli usati e dalle modifiche più o meno consistenti operate da Plauto. Quel che manca a tutt'oggi, però, è un'indagine volta a mettere in rilievo l'importanza del monologo di Periplectomeno su un duplice versante: da una parte, il suo ruolo nell'incarnare il meccanismo del comico su cui si regge l'opera, ovvero lo 'spirito' che la percorre; dall'altra, il suo contributo alla definizione di uno stile di vita -e, implicitamente, di un modello educativo- destinato ad incontrare grande eco in Terenzio e negli autori d'età tardo-repubblicana.

Il presente contributo si propone di riflettere su questi due aspetti osservando le occorrenze del vocabolo lepidus, costantemente associato alla figura di Periplectomeno, nel Miles e non solo. Lo scopo sarà quello di mostrare come sia possibile designare una vera e propria 'ideologia del lepidum' e come questa si evolva nel tempo: se il modello di 'lepiditas'

1 Gli ultimi che hanno cercato di prendere posizione a riguardo sono Paratore 1959, Schaaf 1977, Lefèvre 1984, Zwierlein 1991. Ad essi si rimanda anche per la storia della cosiddetta Kontaminationfrage.

2 Così Saylor 1977, Morenilla 1993, Maurice 2007, sulla linea di quanto, in generale per Plauto, aveva già postulato con forza Barchiesi 1957.

3 Uno, l'A lazón, sarebbe quello dal quale l'autore derivò la beffa principale, giocata ai danni del soldato fanfarone Pirgopolinice; dall'altro, a noi ignoto, sarebbe stato preso il motivo minore dell'inganno al servo Sceledro, che, sebbene non funzionale in senso stretto alla risoluzione della commedia, raddoppia, però, il meccanismo del comico.

5 Saylor 1977. 
rappresentato dal personaggio plautino viene ripreso da Terenzio e risuona ancora in autori quali Cicerone e Catullo, a partire dall'età augustea si assiste ad un declino dell'uso e della gamma di significati del termine, che appare sempre più banalizzato e ridotto alla sola funzione di 'marcatore' del genere comico.

Per prevenire possibili obiezioni, inoltre, -nonché per spiegare la ragione del conio di 'lepiditas' al posto di lepos- è necessario precisare fin da subito che lepidus, pur nella sua varietà di sfumature ${ }^{6}$, è normalmente inteso come tutto ciò che reca piacere secondo due principali categorie semantiche ${ }^{7}$ : la prima è quella di una bellezza puramente esteriore, per cui l'aggettivo viene reso come "grazioso, bello"; la seconda è quella dell'arguzia e della sagacia, da cui derivano traduzioni come "spiritoso, divertente". Nei passi testuali di seguito riportati, soprattutto quelli della prima sezione ('lepiditas' come meccanismo del comico), l'opzione "spiritoso, divertente" non crea nessuna difficoltà, ma la crea il contesto in cui Periplectomeno viene definito come tale, ovvero le sue dichiarazioni, che si riferiscono ad argomenti per niente faceti; in quelli della seconda sezione ('lepiditas' come stile di vita e modello educativo), invece, la traduzione "spiritoso, divertente" è spesso insufficiente e appare preferibile una del tipo "liberale, amabile, accondiscendente", intendendo con ciò non una generica disponibilità, ma una particolare compiacenza nei confronti di una mentalità che potremmo definire 'giovanile'8. È tenendo in considerazione queste accezioni specifiche del termine che si è sentita la necessità del conio di 'lepiditas'. Ed è questo il filo rosso che si vuole seguire nella presente analisi dell' aristia di Periplectomeno e del suo possibile Nachleben.

\section{1. 'Lepiditas' come meccanismo del comico}

È ben noto che il Miles gloriosus, accanto forse al solo Pseudolus, costituisce, nel repertorio plautino, l'opera che meglio incarna il trionfo dell'astuzia del servo. In più occasioni Palestrione si presenta come l'architetto di un piano che gli altri personaggi si dispongono volentieri ad eseguire,

6 Indagate approfonditamente da Monteil 1964, pp. 135-165.

7 Così OLD. Nel $T L L$, invece, non si coglie questa bipartizione, a cui è sostituita quella tra un uso proprio e uno ironico del vocabolo. All'interno di questi due usi vi è un'ulteriore bipartizione a livello dei referenti (esseri umani o cose) ai quali è riferito l'aggettivo.

8 Anche se Monteil 1964, pp. 152-156 individua questa particolare sfumatura di significato di lepidus in quella che ella definisce "l'idée d'un natural plaisant, sociable, serviable" (p. 152), lo studioso si limita poi ad elencare i personaggi che mostrano questa particolare dote senza spiegare il motivo per cui si meritano tale denominazione e senza individuare il nesso vecchi/giovani che sarà centrale nella presente argomentazione. 
riconoscendogli il ruolo indiscusso di leader. Il gioco dell'inganno e della finzione, esibito con fare compiaciuto sul palcoscenico dal servo che orchestra la sua commedia, coinvolge il pubblico in una riflessione sulla natura delle rappresentazioni sceniche e dei loro meccanismi: nota dominante del Miles, dunque, è il suo carattere meditativo, ovvero il suo essere uno degli esempi più chiari di metateatro nell'antichità ${ }^{9}$. Come conseguenza di ciò, non si sbaglierebbe a cogliere nell'esecuzione brillante di un piano organizzato non solo per ingannare, ma soprattutto per divertire e divertirsi ingannando, lo 'spirito' della commedia plautina, il meccanismo su cui si regge il comico. Eduard Fränkel e Marino Barchiesi hanno da lungo tempo individuato in questo aspetto il tratto distintivo di Plauto e la sua originalità nella rielaborazione dei modelli greci, sottolineando come tutte le figure che partecipano al piano del servo, vero alter ego in scena dell'autore, siano 'emanazioni' della sua volontà che si traduce in azione ${ }^{10}$ : tutti i personaggi eseguono la parte che è stata pensata per loro, riproducendo in se stessi l'irresistibile calliditas propria della mente che ha ideato il plot dell'inganno.

Ciò detto, potrebbe sorprendere, allora, che al centro del Miles gloriosus non si trovi un monologo di Palestrione, ma di un suo aiutante, il vecchio vicino di casa Periplectomeno, che dovrebbe essere un mero esecutore delle disposizioni dell'altro. A rafforzare quest'anomalia vi è la constatazione che è proprio Palestrione a fare eco, entusiasta, alle affermazioni di Periplectomeno nella scena dell'aristia; è a lui, per giunta, che il servo lascia il compito di portare a compimento, da trionfatore, sia la prima beffa a Sceledro che quella finale al soldato. Chi è - viene dunque da chiedersi - l'eroe comico della vicenda? Il servus callidus o il senex lepidus?

Nel Miles l'aggettivo lepidus o l'avverbio lepide compaiono per ben trentacinque volte, aggiudicandosi il numero massimo di attestazioni in una commedia plautina. In riferimento a Periplectomeno, il termine è usato quattro volte, e sempre in bocca a Palestrione: due ad introduzione del personaggio (v. 134, quando si racconta che Pleusicle prende alloggio apud suom paternum hospitem, lepidum senem; v. 155, quando il servo vede arrivare il vecchio e commenta "hic illest lepidus quem dixi senem") e due come commento ammirato alle parole del vecchio durante l'aristia (v. 649, o lepidum semisenem; v. 725, o lepidum caput). Del resto, il senex lepidus era una delle possibili varianti del carattere del senex -lo vedremo meglio in seguito- e l'uso di questa espressione quasi formulare in riferimento a

9 Si concentra su quest'aspetto soprattutto Maurice 2007.

10 Vd. Fränkel 1960, pp. 223ss., che vede nell'egemonia del ruolo del servo uno dei tratti più distintivi del "plautino in Plauto", e Barchiesi 1957, pp. 194-196 (p. 196: "Il personaggio è ancora se stesso, ma come sommato ad un altro che si contempla e si descrive e gioca con sé quel ludus che è il ludus del servo, e cioè del poeta stesso"). Su questi temi ritorna Petrone 1977, pp. 10-28. 
Periplectomeno doveva immediatamente servire al pubblico ad inquadrare il personaggio in una determinata categoria.

L'aggettivo, però, non si trova solo in riferimento a Periplectomeno, ma anche per commentare le parole o le azioni di Palestrione, dell'adulescens Pleusicle, della bella Filocomasio, delle due cortigiane Milfidippa e Acroteleuzio, nonché - in chiave ironica - del miles stesso. A loro volta, tutti questi personaggi lo usano ed inseriscono nei propri discorsi. Sembra quasi che in lepidus, dunque, sia racchiusa l'essenza del messaggio che il testo vuole trasmettere, un messaggio che si irradia agli altri personaggi come riflesso della personalità di Periplectomeno, non del servo. Vale la pena, allora, ripercorrere brevemente il contenuto dell'aristia del vecchio, al cui interno, come è ovvio, lepidus compare con particolare frequenza, per arrivare a definire la 'lepiditas' e a capire perché mai questa qualità, dal centro del testo, si propaghi ad illuminare di senso tutte le altre sue parti.

Di fronte agli scrupoli di Pleusicle, che si rammarica di avergli fornito così tante preoccupazioni, Periplectomeno reagisce con veemenza:

Plaut. Mil. 616-634

PL. At hoc me facinus miserum macerat

meumque cor corpusque cruciat. PE. Quid id est quod cruciat? Cedo.

PL. Me tibi istuc aetatis homini facinora puerilia

obicere, neque te decora neque tuis virtutibus;

ea te expetere ex opibus summis mei honoris gratia

mihique amanti ire opitulatum atque ea te facere facinora,

quae istaec aetas fugere facta magi' quam sectari solet:

eam pudet me tibi in senecta obicere sollicitudinem.

PA. Novo modo tu homo amas, siquidem te quicquam quod faxis pudet;

nihil amas, umbra es amantis magi' quam amator, Pleusicles.

PL. Hancine aetatem exercere mei me amoris gratia?

PE. Quid ais tu? Itane tibi ego videor oppido Accherunticus?

Tam capularis? Tamne tibi diu videor vitam vivere?

Nam equidem hau sum annos natus praeter quinquaginta et quattuor,

clare oculis video, pernix sum manibus, pedibus mobilis.

PA. Si albicapillus hic, videtur neutiquam ab ingenio senex.

Inest in hoc emussitata sua sibi ingenua indoles.

PL. Pol id quidem experior ita esse ut praedicas, Palaestrio;

nam benignitas quidem huiius oppido adulescentulist. 
PL. Ma c'è un fatto che mi affligge tremendamente e mi tormenta animo e corpo. PE. Cos'è che ti tormenta? Dimmi! PL. Il coinvolgere te, un uomo della tua età, in queste bravate giovanili, che non sono adatte a te e ai tuoi meriti; che ti prodighi in esse con grande dispendio per amor mio, e che vieni in aiuto a me, a un giovane innamorato, facendo cose che la tua età preferisce evitare che seguire. Provo vergogna a procurarti fastidi nella tua vecchiaia. PA. Ami in un modo nuovo tu se davvero ti vergogni di quello che hai fatto. Tu non ami affatto; sei l'ombra di un amante piuttosto che un amante, Pleusicle. PL. Che io debba affaticare la tua età per i miei amori? PE. Ma che cosa dici? Ti sembro dunque bell'e pronto per andarmene all'altro mondo? Così vicino alla tomba? Pensi che io viva da troppo tempo? Eppure non ho più di cinquantaquattro anni; ci vedo bene con i miei occhi, sono agile di mani, spedito di piedi. PA. Se costui ha i capelli bianchi, per carattere non sembra affatto un vecchio: ha un animo nobile, lavorato a perfezione. PE. Per Polluce! Mi rendo conto che è proprio come dici tu, Palestrione; in effetti la sua affabilità è di certo quella di un giovane.

Se l'adulescens recita con poca convinzione la sua parte di focoso innamorato -e questo gli arreca i rimproveri di Palestrione, che lo chiama "l'ombra di un amante"-, le esuberanti dichiarazioni del senex sono accolte dal servo con grande entusiasmo. Si assiste, dunque, ad un rovesciamento dei ruoli, in cui il giovane fa appello alla gravitas che dovrebbe contraddistinguere gli anziani e si sente in colpa, mentre il vecchio rifiuta di essere considerato tale e vuole dare un'immagine di sé che lo faccia accettare dagli altri come un compagno di bravate, un loro pari. I suoi tentativi hanno successo, perché sia Pleusicle che Palestrione, nel prosieguo dell'autopresentazione del vicino di casa, arrivano progressivamente ad ammettere di trovarsi di fronte ad un senex che senex non è: se in bocca a Palestrione troviamo la definizione riuscitissima di lepidum semisenem ${ }^{11}$ (v. 649), Pleusicle afferma che non è certo possibile trovare un altro senex che sia lepidior (v. 660) e Periplectomeno stesso garantisce che riuscirà a dimostrare di avere i costumi di un adolescente (v. 661, adulescentem moribus). In sintesi, dunque, vi è un graduale smantellamento della maschera del senex a favore di quella dell'adulescens ${ }^{12}$, e tale processo si accompagna all'uso rimarcato di lepidus.

11 Tale lezione è frutto di una congettura alle lezioni tradite semine/semisemne; viene accolta da Lindsay 1905 (di cui si segue l'edizione) e, respinta, invece, da Leo 1896. Si tratterebbe di un hapax, cosa che, però, in Plauto non crea difficoltà, considerata la frequenza dei composti e delle neoformazioni con "semi".

12 Su questo punto si concentra anche Raccanelli 1998, pp. 140-144 nella sua analisi dell'aristia del vecchio. 
L'aristia di Periplectomeno, però, non è ancora terminata, né l'inversione dei ruoli tra lui e Pleusicle ha raggiunto la sua climax. È proprio l'adulescens che si trova a dover difendere i valori tradizionali del matrimonio e dei figli, di cui il senex - scapolo convinto - non vuole sentir parlare ${ }^{13}$ :

\section{Plaut. Mil. 678-683}

PE. Liberae sunt aedis, liber sum autem ego: me volo vivere.

Nam mihi, deum virtute dicam, propter divitias meas

licuit uxorem dotatam genere summo ducere;

sed nolo mi oblatratricem in aedis intro mittere.

PL. Qur non vis? Nam procreare liberos lepidumst opus.

PE. Hercle vero liberum esse tete, id multo lepidiust.

PE. La mia casa è libera, e libero sono io; voglio vivere come mi pare e piace; grazie alle mie ricchezze, per dono degli dèi, mi sarebbe stato possibile prendere in moglie una donna con una ricca dote e dei più alti natali; ma non voglio tirarmi in casa una cagna ululante. PL. Perché non vuoi? È pur bel compito procreare dei figli. PE. Per Ercole! Ma che tu sia libero, questo è molto più bello!

Se Periplectomeno è orgoglioso di non aver preso moglie, Pleusicle cerca di spostare il discorso sul piano dei figli: sposarsi è necessario per avere una discendenza, e questo è, secondo lui, un lepidum onus, "un bel compito". Ma l'altro non accetta una tale definizione, che gli doveva parere ossimorica, e riporta i termini al loro significato proprio: lepidum non è procreare figli (liberi), ma essere liberi (liberum esse); procreare figli è solo un'incombenza, tutto il contrario della libertà. Chiaramente, Palestrione si dichiara d'accordo, ma Pleusicle non demorde:

Plaut. Mil. 701-706

PA. Di tibi propitii sunt, nam hercle si istam semel amiseris libertatem, hau facile in eundem rusum restitues locum. $P L$. At illa laus est, magno in genere et in divitiis maxumis liberos hominem educare, generi monumentum et sibi. $P E$. Quando habeo multos cognatos, quid opu' sit mihi liberis? Nunc bene vivo et fortunate atque ut volo atque animo ut lubet.

13 Vd. a riguardo Lentano 1996, pp. 70-100, che pone Periplectomeno come campione della "paternità rifiutata" e ne indaga i motivi storico-sociali, senza rinunciare a considerare anche le scuole di pensiero filosofiche in cui si potevano riscontrare analoghe forme di rifiuto del matrimonio e della famiglia. 
PA. Gli dèi ti sono favorevoli, perché, una volta perduta codesta libertà, non facilmente la porterai indietro nel medesimo luogo, per Ercole! PL. Ma è un motivo di lode che un uomo di nobile stirpe e in possesso di grandissime ricchezze allevi dei figli, monumento per la sua famiglia e per il suo nome. PE. Visto che ho tanti parenti, che bisogno ho di figli? lo vivo bene e felicemente adesso e come mi pare e piace.

In questo passaggio le parole del giovane suonano proprio come quelle di un anziano pater familias (tanto è vero che, poco dopo, al v. 751, Periplectomeno lo inviterà a lasciare istanc orationem hinc veterem atque antiquam), mentre il vicino di casa continua la sua perorazione: avere figli non è necessario (quid opu'sit), perché un uomo ricco e benvoluto come lui è circondato da amici e parenti disposti a prendersi cura della sua persona e, soprattutto, delle sue sostanze. Essere padre, invece, comporterebbe solo uno stato di continua apprensione:

Plaut. Mil. 719-725

PE. Pol si [scil. filios] habuissem, sati' cepissem miseriarum e liberis: continuo excruciarer animi: si ei fort' fuisset febris, censerem emori; cecidissetve ebrius aut de equo uspiam, metuerem ne ibi diffregisset crura aut cervices sibi.

PL. Huic homini dignum est divitias esse et diu vitam dari, qui et rem servat et se bene habet suisque amicis usui est. PA. O lepidum caput!

PE. Per Polluce! Se avessi avuto dei figli, ne avrei avute abbastanza di preoccupazioni! Sarei stato continuamente in ansia: se uno di loro si fosse preso la febbre, avrei pensato che stava per morire; se fosse caduto da qualche parte, ubriaco, o smontando da cavallo, avrei temuto che si fosse spezzato una gamba o l'osso del collo. PL. È giusto che quest'uomo sia ricco e che gli sia concessa una lunga vita, lui che conserva i suoi beni, se la passa bene ed è utile ai suoi amici. PA. Che uomo di spirito!

Qui Periplectomeno sembra fare il verso ai padri della commedia, o meglio, lo completa: se prima aveva dipinto la schiavitù a cui è sottoposto chi si prende in moglie una uxor dotata -situazione a cui il pubblico del dramma doveva aver assistito molte volte nel corso di altre commedie-, ora descrive i crucci dei tanti patres sempre in pena per il destino dei figli di cui è pieno il teatro antico ${ }^{14}$.

14 Vd. Minois 1988, p. 107: il ritratto di Periplectomeno "è di fatto in negativo quello del vecchio comune, la cui condotta è fustigata una volta di più". 
Quest'ultima dichiarazione di indipendenza nei confronti del ruolo che tradizionalmente un senex agiato come lui avrebbe dovuto giocare nella vita e sul palcoscenico provoca l'ammirazione entusiasta e definitiva di Palestrione, che, dopo avergli ancora attribuito la denominazione di lepidus, lo prende ad esempio di un nuovo e rivoluzionario modello di probitas ${ }^{15}$, degno di essere premiato dagli dèi (vv. 725-735). E così - senza contare una piccola coda riguardante ulteriori scrupoli di Pleusicle sui costi arrecati al vicino di casa, prontamente respinti dal vecchio - si conclude l'aristia di Periplectomeno.

La vicenda riprende poi il suo corso e Periplectomeno, per quanto coprotagonista essenziale dello svolgimento del piano, sembrerebbe messo in ombra dall'astuzia di Palestrione e dalla spassosa comicità che la beffa orchestrata contro Pirgopolinice produce. Eppure, come si è già accennato poco sopra, l'impiego di lepidus nel testo non solo continua, ma diventa addirittura martellante: ogni volta che si parla del tranello orchestrato dal servo, o di qualche altra trovata brillante, il termine non tarda a comparire; ogni volta che qualche personaggio esegue bene la sua parte, la parola di lode che ne sottolinea l'operato è sempre quella che ha accompagnato fin dalla sua entrata in scena il senex. La commedia si chiude, addirittura, nel nome della 'lepiditas', e con ironia: in 4.9, quando il miles è invitato ad entrare nella casa di Periplectomeno, dove - non a caso - avrà luogo la beffa finale e la punizione definitiva, uno schiavetto lo saluta con l'apostrofe vir lepidissume (v. 1382). All'apice della beffa, lepidus non può che trovarsi al grado superlativo, riferito con scherno al personaggio la cui rovina è stata il bersaglio della 'lepiditas' di tutti gli altri.

Ritornando alla domanda di partenza, dunque, e dopo aver ripercorso i passi dell'aristia di Periplectomeno in cui compare lepidus, pare possibile avanzare un'ipotesi circa il carattere saliente del Miles, la sua vena comica dominante. Periplectomeno incarna lo stile di vita di chi ha scelto di vivere la propria esistenza all'insegna di un ludus che non si esaurisce nell'arguzia ingegnosa del servus callidus, ma che diventa una condizione permanente e un modello di comportamento. Nella figura del senex che trasgredisce le regole non perché è giovane e innamorato, ma quasi per partito preso, la commedia plautina trova il suo eroe, un eroe superiore al servo stesso, perché non conosce e non si dà limiti temporali. La commedia, normalmente, è lo spazio privilegiato del carnevalesco bachtiniano, il luogo in cui è concesso trasgredire alle norme sociali purché alla fine tutto torni a ristabilirne l'ordine ${ }^{16}$. In un contesto del genere, il servo mette a servizio della condotta sregolata e imprudente del giovane la sua intelligenza,

$\overline{15}$ Sulla creazione di una vera e propria moralità rovesciata plautina, vd. Labate 2012.

16 Il concetto è largamente esplorato. Per quanto riguarda Plauto, si rimanda qui solo a Petrone 1977, pp. 25-28, Bettini 1981 e Lentano 1996, pp. 165-170. 
ma il gioco sarà di breve durata: l'adulescens non sposerà la cortigiana, a meno che la sorte non collabori a farne scoprire i liberi natali. Nel Miles, invece, abbiamo un senex che è rimasto giovane per sempre, ed è questo che lo rende spiritoso e divertente, che gli conferisce, insomma, l'appellativo di lepidus. Ma la sua 'lepiditas' si riverbera su tutti gli altri personaggi e, in definitiva, sulla commedia stessa, perché vi si celebra una trasgressione che pare non conoscere i confini né del dramma né della vita: alla fine, Pleusicle si porta via la sua bella, senza che venga minimamente accennato alle difficoltà di un matrimonio, senza che nessuno della famiglia si opponga a questo amore fuori dalle regole, senza che, in sostanza, il pubblico si senta rassicurato dal ritorno alla normalità ${ }^{17}$. L'eccezionalità dello stile di vita di Periplectomeno diventa l'eccezionalità dell'intera commedia, l'unica in cui la riuscita dell'intrigo del servo non deve fare i conti con la realtà e le sue convenzioni, ma ne sancisce una perenne sospensione. Il Miles gloriosus è una commedia lepida perché celebra il trionfo utopico di un'eterna ribellione, di un'eterna giovinezza ${ }^{18}$. E Periplectomeno, con la sua aristia, ne è il campione.

\section{2. 'Lepiditas' come stile di vita e modello educativo}

Nel suo studio dedicato all'analisi dei vari tipi di personaggi che compaiono nella commedia latina, George E. Duckworth ${ }^{19}$ nota come la figura del senex, seconda per numero di comparse nell'opera plautina solo a quella del servo ${ }^{20}$, possa suddividersi in tre categorie: il vecchio pater, che può essere sia severo ma credulone, e in quanto tale facilmente gabbato dal servo, sia favorevole ad un'educazione più liberale nei confronti dei figli; il vecchio amator, non di rado un padre inflessibile che si trasforma in gaudente libertino e rivale del figlio, normalmente esposto al ridicolo; il vecchio amicus, che di solito veste i panni di aiutante del senex padre in difficoltà. Lo studioso inserisce Periplectomeno all'interno della terza categoria, ma una simile collocazione crea non poche difficoltà. In primo luogo,

$\overline{17}$ Non è un caso che Bettini 1982 inizi la sua analisi delle strutture essenziali delle commedie plautine proprio dal Miles gloriosus (pp. 43-47): esso rappresenta, infatti, il caso più lineare del passaggio di un bene $\mathrm{C}$ (in questo caso la cortigiana) da $\mathrm{B}$ che lo detiene (il miles) ad A che lo reclama 'illegalmente' e lo sottrae con l'inganno (il giovane Pleusicle e i suoi aiutanti). Impedimenti familiari o interventi della sorte non sopraggiungono a complicare la situazione.

18 Non si può che convenire con il titolo di Bettini 1981, "un'utopia per burla": il ludus plautino, che porta sulla scena la trasgressione, finisce per essere un'utopia di un altro mondo senza volerlo, ossia solo per l'estremo esercizio della fantasia e del gioco dell'intelligenza.

19 Duckworth, pp. 242-249.

20 Vd. Minarini 1995, p. 1: nelle commedie di Plauto il senex appare per un totale di 30 volte contro le 36 del servus. 
infatti, Periplectomeno non aiuta un suo coetaneo, ma si mette a disposizione di un giovane, Pleusicle, e del suo schiavo; in secondo luogo, pur non ostacolando in alcun modo ma anzi aiutando la felice riuscita del piano di Palestrione, Periplectomeno fornisce una descrizione di sé che per alcuni aspetti lo avvicina ai senes libertini, il cui comportamento è generalmente disapprovato dall'autore e dal pubblico ${ }^{21}$. La condiscendenza nei confronti delle follie della gioventù, infine, lo avvicina alla figura del pater liberale.

Come si è visto, però, la scelta di non sposarsi e di non avere figli lo rende un caso eccezionale, dal momento che i senes plautini sono praticamente sempre anche dei mariti e dei padri. L'ibrido statuto del personaggio ha già suscitato l'interesse di parecchi studiosi ${ }^{22}$, tra i quali vi è chi ha posto l'accento sui versi che descrivono Periplectomeno come intrattenitore gaudente ed amante dei banchetti per dedurre una rappresentazione caricaturale del personaggio, che sarebbe, accanto al miles stesso, bersaglio degli strali plautini ${ }^{23}$. Non mi sembra che il ruolo essenziale rivestito dal senex all'interno della commedia, investigato fin qui, possa andare di pari passo con la demolizione dello stesso; al massimo, si può dire che al lato 'godereccio' di Periplectomeno sia dato risalto per strappare al pubblico qualche risata, ma si tratta sempre di una risata ammirata, mai di derisione. Più importante di questi elementi più spassosamente comici dell'aristia, è, invece, la caratterizzazione di Periplectomeno come lepidus, e, questa volta, in un'accezione del termine un po' diversa da quella esplorata fin qui.

La domanda di partenza non può che essere questa: oltre a Periplectomeno, chi sono gli altri senes lepidi delle commedie plautine e a quale delle tre categorie sopra delineate appartengono ${ }^{24}$ ? Propriamente parlando, vi è solo un altro personaggio a cui viene data la definizione di senex lepidus: Callifone nello Pseudolus. Costui, vecchio amico di Simone, padre di Calidoro, mostra indulgenza nei confronti del giovane e, al contrario di Simone, che impersona il padre dai severi costumi, rammenta all'amico le pazzie fatte in gioventù. Il suo comportamento gli attira l'ammirazione del servo, che, similmente a quanto abbiamo visto nel Miles, 'battezza' Callifone senex lepidus, ed esclama che tutti così dovrebbero essere i padri con i figli:

Plaut. Pseud. 433-444

CA. [...] Sed si sint ea vera, ut nunc mos est, maxume quid mirum fecit, quid novom, adulescens homo

\footnotetext{
${ }^{21}$ Per un quadro complessivo dei senes amatores in Plauto, vd. Ryder 1984, che cita Periplectomeno come figura ai margini, che proprio la caratterizzazione positiva di lepidus esclude dall'elenco degli amatores, e Minarini 1995, pp. 8-13.

22 Vd. Raccanelli 1998, pp. 140-144 e Morenilla 1993.

23 Vd. Morenilla 1993, pp. 80-94.

24 Una carrellata dei senes lepidi in Minarini 1995, pp. 18-20.
} 
si amat, si amicam liberat? PS. Lepidum senem!

SI. Vetus nolo faciat. CA. At enim nequiquam nevis;

vel tu ne faceres tale in adulescentia.

Probum patrem esse oportet, qui gnatum suum

esse probriorem quam ipsus fuerit postulet.

Nam tu quod damni et quod fecisti flagiti

populo viritim potuit dispertirier.

Idne tu mirare, si patrissat filius?

PS. ${ }^{\Omega} \mathrm{Z} \varepsilon \tilde{v}$, quam pauci estis homines commodi! Em,

illic est pater, patrem esse ut aequom est filio.

CA. Ma anche se tutte queste cose fossero vere, come è il costume di oggi, che cosa mai ha fatto di assolutamente straordinario, di nuovo, un giovane, se è innamorato, se affranca la sua amante? PS. Che vecchietto di larghe vedute! SI. Non voglio che faccia una cosa risaputa. CA. Ma non lo vuoi invano; oppure non avresti dovuto fare lo stesso nel corso della tua giovinezza. Ė necessario che sia un padre probo, colui che pretende che suo figlio sia più probo di quanto era stato lui. Infatti sarebbe appena possibile distribuire al popolo tutti i danni e le scelleratezze che hai fatto. E ora ti meravigli, se il figlio assomiglia al padre? PS. o Zeus, che pochi siete voi uomini accondiscendenti! Quello è un padre tale quale è giusto che un padre sia con il figlio.

La figura di Callifone, però, al confronto con quella di Periplectomeno, dà l'impressione di essere mancata, non sfruttata in tutte le sue potenzialità: l'assistenza e l'aiuto del vecchio compiacente, infatti, non saranno mai richiesti nel prosieguo della commedia e Pseudolo trionferà da solo. In più, egli rimane in primo luogo l'amico di un senex, e gli rammenta moderazione ed indulgenza in ricordo delle bravate commesse in gioventù: questo ci lascia intuire che ora non ne fa più. Di certo, però, egli rimane l'unico non-padre, accanto a Periplectomeno, a essere chiamato quasi in modo formulare senex lepidus, mentre gli altri senes a cui sia attribuita la qualifica di lepidi sono patres che possono raggiungere o perdere questo status a seconda di come regolano il loro rapporto con i figli. Tra gli esempi più significativi, vale la pena di citare quello di Demeneto nell'Asinaria e di Filtone nel Trinummus.

Demeneto sembrerebbe un padre liberale che cerca di instaurare con il figlio un rapporto di alleanza e complicità. Pur essendosi sposato, egli ha in comune con Periplectomeno la disponibilità ad aiutare i giovani nei loro intrighi. L'analogia diventa esplicita quando il servo Leonida, ricordando come il vecchio gli avesse tenuto il gioco mentre orchestrava la sua beffa, lo chiama lepidus: Asin. 580-581, edepol senem Demaenetum lepidum 
fuisse nobis. Ut adsimulabat Sauream me<d> esse quam facete! ("Come è stato disponibile il vecchio Demeneto con noi. Con quanta maestria faceva finta che io fossi Saurea!"). Anche in questo caso, dunque, il riconoscimento della 'lepiditas' avviene ad opera del servus callidus; la partecipazione attiva del senex al tranello, inoltre, lo rende effettivamente la figura più simile a Periplectomeno nell'orizzonte delle commedie plautine. Ad un certo punto, però, Demeneto cambia improvvisamente. A piano riuscito, quando il figlio si appresta a godere le gioie dell'amore, il padre gli impone una dura condizione: una notte d'amore con la sua bella. La presunta complicità che aveva sempre millantato non era altro, in realtà, che il desiderio di poter defraudare il giovane amator dei suoi diritti. Ostacolando la felicità della coppia protagonista, Demeneto passa dal ruolo di aiutante a quello di antagonista, e questa rapida transizione lo porta a perdere lo status di senex lepidus. D'ora in avanti egli verrà tratteggiato con le sembianze denigranti del senex amator che, in quanto tale, sarà punito e beffato al termine della commedia. Se Periplectomeno, al contrario, mantiene la sua 'lepiditas' dall'inizio alla fine, questo avviene perché impersona senza cedimenti il ruolo di campione di liberalità nei confronti dei giovani, e senza fini personali.

Anche nel Trinummus la figura del vecchio padre subisce una metamorfosi, ma di segno opposto rispetto a quella di Demeneto, e non irreversibile. Padre liberale all'inizio, Filtone si trasforma in un pater austero quando si rende conto che il figlio Lisitele vorrebbe sposare una donna senza dote. Alla fine, però, dopo essersi lasciato convincere ad andare contro la salvaguardia del buon nome e delle sostanze della famiglia, acconsente ai desideri del figlio, che così lo loda:

Plaut. Trin. 378-390

PH. Egone indotatam te uxorem ut patiar?

LY. Patiundumst, pater;

et eo pacto addideris nostrae lepidam famam familiae.

PH. Multa ego possum docta dicta et quamvis facunde loqui;

historiam veterem atque antiquam haec mea senectus sustinet.

Verum ego, quando te et amicitiam et gratiam in nostram domum

video adlicere, etsi adversatus tibi fui, istac iudico.

Tibi permitto, posce, duce.

LY. Di te servassint mihi!

[...]

Lepidus vivis.

FI. E io dovrei tollerare che tu prenda una moglie senza dote? LI. Lo devi tollerare, padre. E così avrai conferito una fama di liberalità alla 
nostra famiglia. FI. Potrei citare molte sagge massime ed esprimermi in modo sommamente eloquente: la mia vecchia vita conserva il ricordo di una storia antica e dei tempi andati. Ma dal momento che vedo che vuoi portare alla nostra casa amicizia e gratitudine, anche se prima ti ho avversato, questo ora stabilisco. Te lo concedo, chiedila, prendila in sposa. LI. Gli dèi mi ti conservino! Ti comporti liberalmente.

Lepidi, quindi, si nasce, ma ci si può anche dimenticare di esserlo, o lo si può diventare. Se la perdita di questo status, nel Trinummus, scatena il conflitto generazionale, la transizione alla 'lepiditas' ne segna la risoluzione e rende possibile il lieto fine della commedia. Laddove, invece, come nell'Asinaria, il rapporto di collaborazione padre-figlio si rivela rovinato per sempre, il lieto fine non si può che imporre attraverso la beffa e la ridicolizzazione della figura anziana.

Questo ci conduce ad alcune importanti conclusioni. In primo luogo, il senex lepidus percorre trasversalmente le tre categorie di senes individuate da Duckworth - che sono, di per se stesse, mobili -, ma la caratteristica che fa di uno di loro un personaggio degno di meritare l'appellativo di lepidus è sempre una sola, ovvero la condiscendenza nei confronti dei desideri dei giovani, l'essere moderni, contrari agli eccessivi rigori del mos maiorum. Tale condiscendenza rende i senes lepidi i rappresentanti di un modello educativo fondato sulla comprensione e la disponibilità che doveva incontrare una particolare risonanza nella Roma che si preparava agli scontri tra Catone il Censore e il circolo degli Scipioni.

È indubbio, però, che definire la 'lepiditas' un modello educativo faccia emergere ulteriormente l'eccezionalità di Periplectomeno, e la problematicità della sua posizione: egli, infatti, è sicuramente lepidus in quanto aperto nei confronti dei giovani, ma questa è la conseguenza del suo anomalo sentirsi adulescens, dell'aver rifiutato il peso della paternità. Proprio per questo il Miles gloriosus, pur rappresentando il trionfo di una 'lepiditas' che si impone dall'inizio alla fine del dramma, fornisce anche le basi, però, della sua debolezza come modello educativo: è facile essere lepidi come Periplectomeno, quando non si hanno figli; è facile essere accondiscendenti con i figli degli altri.

Allargando le riflessioni iniziate poco sopra, dunque, si può dire che il Miles rappresenti l'unica commedia plautina che non mette in scena né padri né conflitti generazionali, ma mostra un mondo impossibile, lontano da casa e 'sradicato', dove gli unici vecchi sono scapoli, eternamente giovani e amici dei giovani, ed il riso viene generato solo dalla beffa ai danni di un bersaglio esterno, senza che sia implicato uno scontro all'interno di un nucleo familiare: è un mondo di giovani per i giovani, ed è per questo che il lepidum trionfa. Già nell'Asinaria e nel Trinummus, come abbiamo 
visto, la situazione si complica, diventa più problematica, perché il senex lepidus è un padre, e non sempre riesce a mantenersi fedele ai principi di liberalità che professa. Per capire se un modello educativo fondato sulla 'lepiditas' possa funzionare oppure no, in sostanza, è necessario che siano i padri ad usarlo, ed è qui che interviene Terenzio.

\section{La 'lepiditas': un modello vincente?}

Il problema del rapporto padre-figlio, toccato solo di striscio da Plauto, è, come è noto, il cuore del dramma terenziano ${ }^{25}$, e della commedia Adelphoi in particolare ${ }^{26}$. Gli opposti metodi educativi dei due fratelli Micione e Demea - il primo, assertore convinto di una generosa apertura nei confronti dei misfatti della gioventù; il secondo, rigoroso e severo seguace dei dettami del mos maiorum - vengono indagati da Terenzio con uno spirito critico capace di passare al vaglio, di ognuno, e alla prova dei fatti, pregi e difetti.

$\grave{E}$ inevitabile, di conseguenza, che la commedia riproponga la figura del senex lepidus, apparentemente rappresentato da Micione. Costui, anzi, pare essere una versione 'smorzata' di Periplectomeno, a cui spesso è stato accostato ${ }^{27}$. Proprio ad apertura di commedia, infatti, egli tiene un monologo che si avvicina per alcuni aspetti a quello del senex lepidus di Plauto, non solo nei concetti, ma - a volte - in alcuni richiami formali. Rimasto scapolo e senza progenie per scelta, Micione avrebbe condotto la sua vita di agi cittadini per sempre, se non si fosse ritrovato ad educare al posto del fratello Demea uno dei figli di lui, Eschine, di cui decide di diventare confidente ed alleato. Una volta ritrovatosi nel ruolo di padre, Micione sperimenta di persona quelle ansie che lo stesso Periplectomeno aveva previsto nel caso avesse avuto un figlio, come il timore, quando Eschine non è ancora tornato a casa, che abbia preso freddo, sia caduto da qualche parte o si sia rotto qualcosa (vv. 36-37, ne aut ille alserit aut uspiam ceciderit aut praefigerit aliquid). Ciononostante, egli ha sempre incoraggiato le confidenze del nipote e perdonato i suoi eccessi di gioventù, nella convinzione che si possa spingere i figli all'obbedienza grazie ad un metodo educativo liberale piuttosto che con la paura di una punizione (vv. 57-58, pudore et liberalitate liberos / retinere satius esse credo quam metu). Successivamente, in una serie di confronti/scontri con Demea, emerge un altro tratto della personalità di Micione che lo accosta a Periplectomeno, ovvero l'estrema liberalità

25 Vd. Minarini 1995, pp. 26-30.

26 La bibliografia a riguardo è molto vasta. Si citano qui solo Fantham 1971, Tränkle 1972, Lieberg 1988, Eyben 1991, Lentano 1996, Klowski 2000, Citroni Marchetti 2004.

27 Vd. Lentano 1996, pp. 81-100, che vede in entrambe le figure una rottura con la tradizione catoniana del matrimonio e della discendenza come modo tradizionale di accumulare, gestire e trasmettere patrimonio nella società romana. 
e la prodigalità con cui mette a disposizione le sue sostanze per finanziare gli spassi dei nipoti. Si potrebbe concludere, quindi, che egli è quale Periplectomeno sarebbe stato se avesse adottato un figlio?

Le cose non sono così semplici. Micione, certo, non si oppone agli amori avventurosi e alle richieste di denaro di Eschine, ma non approva questo comportamento né tantomeno lo segue. La sua condiscendenza si basa sul presupposto che si tratti di vizi passeggeri, propri dell'età (v. 53, quae fert adulescentia), e che prima o poi il giovane si calmi e 'metta la testa a posto':

Ter. Ad. $100-110$

MI. Quia tu, Demea, haec male iudicas.

Non est flagitium, mihi crede, adulescentulum

scortari neque potare: non est; neque fores

effringere. Haec si neque ego neque tu fecimus, non siit egestas facere nos. Tu nunc tibi id laudi ducis quod tum fecisti inopia? Iniuriumst; nam si esset unde id fieret, faceremus. Et tu illum tuom, si esses homo, sineres nunc facere dum per aetatem decet potius quam, ubi te exspectatum eiecisset foras, alieniore aetate post faceret tamen.

MI. Perché tu, Demea, giudichi male queste cose. Non è una sciagura, credimi, che un ragazzo vada a donne o beva; non lo è; né sfondare una porta. Se né io né tu l'abbiamo fatto, la povertà non ha permesso che lo facessimo. E ora tu ti arrechi a motivo di lode quello che allora hai fatto per miseria? Non è giusto. Perché se ci fosse stato il modo per farlo, lo avremmo fatto. E tu se fossi un uomo, a tuo figlio dovresti permettere che lo faccia ora, finché è lecito per la sua giovane età, piuttosto che, quando finalmente ti abbia messo alla porta, non lo faccia poi ugualmente in età meno opportuna.

Nelle sue parole si ha una chiara distinzione tra ciò che è concesso quando si è giovani e ciò che non è più concesso ad una certa età: certe azioni si possono scusare solo per una licenza a tempo determinato, esattamente come, nel carnevalesco proprio del teatro, la rottura delle norme non è che una sospensione provvisoria delle stesse. Nell'accenno a quell' "età meno opportuna" sembra quasi di poter scorgere una frecciatina a figure quali Periplectomeno stesso. A conferma di ciò, quando apprende che il figlio ha sottratto ad un lenone una cortigiana - esattamente la stessa azione a cui partecipa entusiasticamente Periplectomeno nel Miles - Micio- 
ne non può nascondere un po' di delusione: qualche tempo prima Eschine gli aveva annunciato che avrebbe preso moglie ed egli aveva sperato che i fervori della giovinezza si fossero finalmente acquietati (v. 152, sperabam iam defervisse adulescentiam). Dal momento che così non è, Micione si appresta ad aspettare ancora, con pazienza ed indulgenza. Un pater liberale lo è certamente; che sia anche lepidus, è un'altra questione, e, del resto, questo aggettivo non compare mai in riferimento a lui, ma viene usato, a sorpresa, per lodare l'operato del fratello, il padre scorbutico e tradizionalista.

Dopo un monologo che pare una vera e propria capitolazione, Demea decide di lasciar perdere i suoi metodi educativi e adottare quelli di Micione. Detto fatto, inizia la sua trasformazione: non solo non si oppone al matrimonio di Eschine con la vicina di casa senza dote, ma propone di accelerare il trasferimento di lei in casa abbattendo il muro che separa le due abitazioni; quindi concede la libertà al servo Siro, che aveva aiutato il figlio in tutte le sue bravate. In ambo i casi, è da notare la reazione che accompagna le generose dichiarazioni di Demea:

Ter. Ad. 906-914

DE. Missa haec face, hymenaeum, turbas, lampadas, tibicinas, atque hanc in horto maceriam iube dirui quantum potest: hac transfer: unam fac domum; transduce et matrem et familiam omnem ad nos. AE. Placet, pater lepidissime! DE. Euge! lam lepidus vocor.

Fratri aedes fient perviae, turbam domum adducet, et sumptu amittet multa: quid mea? Ego lepidus ineo gratiam.

DE. Lascia perdere queste cose, l'imeneo, il corteo, le fiaccole, le flautiste, e ordina che questo muro in giardino venga abbattuto il prima possibile. Conducila di là, fa' una sola casa: trasferisci da noi anche la madre e tutta la servitù. ES. Va bene, babbo adorabilissimo! DE. Evviva, già sono chiamato adorabile! La casa di mio fratello sarà aperta a chiunque, si porterà in casa una folla di persone, e per la spesa perderà molti soldi: ma a me che importa? Io facendo l'indulgente entro nelle grazie di tutti.

Ter. Ad. 958-966

SY. Factumst quod iussisti, Demea.

DE. Frugi homo's. Ergo edepol hodie, mea quidem sententia, iudico Syrum fieri esse aequom liberum. MI. Istunc liberum? Quodnam ob factum? DE. Multa. SY. O noster Demea, edepol vir bonu's. Ego istos vobis usque a pueris curavi ambo sedulo: 
docui, monui, bene praecepi semper quae potui omnia.

DE. Res apparet. Et quidem porro haec, obsonare cum fide, scortum adducere, adparare de die convivium:

non mediocris hominis haec sunt officia. SY. O lepidum caput!

SI. È stato fatto quello che hai ordinato, Demea. DE. Sei un uomo onesto. E per questo oggi, a mio parere, ritengo che sia giusto che Siro diventi libero. MI. Costui libero? E per quale ragione? DE. Molti. SI. O caro Demea, sei veramente un uomo generoso. Vi ho curato entrambi costoro assiduamente fin da quando erano bambini: li ho educati, ammoniti, ho sempre insegnato loro tutto quello che ho potuto. DE. E si vede! E certamente anche questo hai insegnato loro, a gozzovigliare con impegno, a frequentare sgualdrine, a organizzare banchetti di giorno: questi non sono compiti di un uomo mediocre. SI. Che uomo di spirito!

Lepidus, raro in Terenzio ${ }^{28}$, compare con forza a sottolineare il cambiamento di Demea, un cambiamento del tutto voluto e pianificato secondo un modello di liberalità che non si vuole imitare, ma piuttosto, portare all'eccesso e ridicolizzare. Lepidus, infatti, non si ritrova solo negli euforici ed ingenui ringraziamenti di Eschine e Siro, ma anche in bocca al senex stesso, che constata soddisfatto iam lepidus vocor e, poco dopo, ego lepidus ineo gratiam. Si ha l'impressione che il termine sia usato in modo programmatico, come etichetta da apporre ad un personaggio e renderlo riconoscibile al pubblico: diventa lepidus non solo chi comprende i desideri della gioventù e li asseconda, ma chi vi prende parte sconsideratamente, chi si atteggia a giovane, chi non progetta il domani. Dietro questa parodia di Micione - che, infatti, pur nelle sue grandi concessioni, non era mai stato chiamato lepidus - il vero bersaglio di Terenzio è Periplectomeno. Micione, lo si è visto, per quanto esageratamente comprensivo, accetta a posteriori le bravate del figlio, nell'illusione che tutto col tempo si sistemi, o rassegnandosi a ciò che ormai non si può più riparare, ma senza prendere parte attivamente ai piani di Eschine e Siro. Le proposte di Demea, invece, non solo accettano lo status quo, ma danneggiano il fratello economicamente e lo costringono ad un matrimonio a tarda età, decisamente fuori tempo, come se fosse un adulescens (novus maritus, commenta irritato Micione al v. 938). Demea non si limita a fare l'amico dei giovani, ma ragiona da giovane, proponendo di abbattere muri, dilapidare soldi, organizzare matrimoni sconvenienti: inizia, insomma, a fare il Periplectomeno di turno. Il plauso è immediato: e non sembra casuale che l'espressione o lepidum caput, che consacra l'avvenuta 'trasformazione', sia posta in bocca al ser-

${ }^{28}$ Ha solo 9 attestazioni contro le 88 di Plauto (vd. Monteil 1964, p. 136). 
vo, e proprio dopo che Demea ne ha approvato gli eccessi. Gozzovigliare con impegno, frequentare sgualdrine, organizzare banchetti di giorno: è un ottimo riassunto del ritratto che Periplectomeno dà di sé nella sua aristia.

Chiaramente, l'approvazione di Demea è ironica, e fasulla anche la sua metamorfosi. Lo si capisce bene al termine della commedia, quando un Micione stupefatto chiede ragione al fratello di un cambiamento tanto improvviso e Demea gli risponde di essere stato così smoderatamente prodigo solo per mostrare come sia facile essere benvoluti senza che questo corrisponda ad un modo saggio e retto di vivere. Concede all'altro figlio, Demifone, che si tenga la sua cortigiana, ma esige che questa sia l'ultima sregolatezza, come se alla fine il metodo educativo proposto da Terenzio sia uno capace di mediare tra eccessiva severità ed eccessiva indulgenza ${ }^{29}$. Su questo punto la critica, negli anni, si è divisa, schierandosi soprattutto 'dalla parte di Demea' 30 , ma non è questo l'aspetto che interessa in questa sede. Notevole, piuttosto, è il dialogo a distanza che il testo di Terenzio sembra intrattenere con quello di Plauto ${ }^{31}$ : un comportamento come quello di Periplectomeno è sicuramente fallimentare, e la sua 'lepiditas', che si mantiene senza cedimenti dall'inizio alla fine della commedia, può funzionare solo all'interno di una commedia come il Miles, che non conosce strutture familiari e le rinnega in nome di un eterno divertimento. Un senex lepidus è un'entità ossimorica nella sua stessa denominazione, una figura che si muove ai confini dell'assurdo, in un ludus senza fine, e che si sgretola al contatto con la realtà: nessun padre si potrebbe davvero comportare così, e la parodia di Demea ne è la dimostrazione più evidente.

\section{Dai senes lepidi ai pueri lepidi: la 'lepiditas' in Cicerone e Catullo}

Numerosissimi studi hanno già indagato la forza del conflitto generazionale scatenatosi negli ultimi anni della repubblica, e le ragioni che lo determinarono: la gioventù romana, estromessa dall'esercizio del potere, reagì alla propria marginalizzazione o ripudiando la politica o, all'opposto, unendosi alle fila di chi voleva rovesciare i vecchi ceti dirigenti ${ }^{32}$. Al primo

29 Così la pensano Duckworth p. 287 e Minarini 1995, pp. 28-30.

30

Come nota Leigh 2004a, pp. 166 e 190-191, che a p. 166, n. 43 elenca i principali studi che sposano questa tesi. Ad essi vanno aggiunti Lentano 1996, pp. 138-139 e Citroni Marchetti 2004, pp. 11-17 e 58-59.

31 Ciò, tra l'altro, depone a favore dell'autenticità plautina dell' excursus su Periplectomeno che, al di là della sua presenza o meno nel modello greco, si rivela essere frutto dell'ingegno di Plauto e non ascrivibile a una successiva aggiunta da parte di un imitatore plautino, come vorrebbe Zwierlein 1991.

32 Per un'ottima sintesi della situazione, vd. Narducci 1989. Per una trattazione più ampia del problema, vd. Neraudau 1979 e Bonnefond 1983, pp. 249-377 (in particolare, pp. 361-368). 
gruppo va ascritta l'attività di Catullo e della cerchia dei poetae novi; al secondo, la militanza di coloro che aderirono alla congiura di Catilina. Contro entrambi si schierò Cicerone, critico severo di una degenerazione dei costumi che, in un senso o nell'altro, pareva dimentica dei valori del mos maiorum. In un'unica famosa orazione, però, la Pro Caelio, egli si dichiara sorprendentemente a favore di un'apertura nei confronti delle nuove generazioni, e lo fa servendosi del mondo e delle maschere della commedia.

Non è necessario ripercorrere nel dettaglio ciò che i contributi di Emanuele Narducci e Matthew Leigh ${ }^{33}$, tra gli altri $^{34}$, hanno già messo ottimamente in luce. Siano sufficienti qui poche parole di sintesi, che servono ad introdurre il contesto in cui si muoverà il nostro discorso. Per smontare le accuse degli avversari, Cicerone, come è noto, rappresenta Celio quale un adulescens irretito dalle grazie di una cortigiana gelosa e senza scrupoli, Clodia. Pur avendo commesso alcuni errori dovuti all'intemperanza dell'età, Cicerone è pronto a scusare il giovane, ritornato ormai sulla retta via, e, per avvalorare la sua posizione, cita nientemeno che il Micione di Terenzio, la cui liberalità e condiscendenza sono contrapposte all'inflessibile rigore dei patres ferrei delle commedie di Cecilio Stazio (Cael. 37-38) ${ }^{35}$. Queste le parole con cui più chiaramente l'oratore espone il metodo educativo che ritiene il più opportuno da adottare con la gioventù moderna:

Cic. Cael. 42

[...] Detur aliqui ludus aetati; sit adulescentia liberior; non omnia voluptatibus denegentur; non semper superet vera illa et derecta ratio; vincat aliquando cupiditas voluptasque rationem, dum modo illa in hoc genere praescriptio moderatioque teneatur. Parcat iuventus pudicitiae suae, ne spoliet alienam, ne effundat patrimonium, ne faenore trucidetur, ne incurrat in alterius domum atque familiam, ne probrum castis, labem integris, infamiam bonis inferat, ne quem vi terreat, ne intersit insidiis, scelere careat. Postremo cum paruerit voluptatibus, dederit aliquid temporis ad ludum aetatis atque ad inanis hasce adulescentiae cupiditates, revocet se aliquando ad curam rei domesticae, rei forensis reique publicae, ut ea quae ratione antea non perspexerat satietate abiecisse et experiendo contempsisse videatur.

\footnotetext{
33 Vd. Narducci 1989 (e la sintesi fornita in Narducci 2009) e Leigh 2004b.

34 Si rimanda a Leigh 2004b per un quadro degli studi riguardanti la Pro Caelio e la commedia. Si cita qui solo Geffcken 1973, primo studio a sviluppare approfonditamente il tema.

35 Si rimanda a May 1995 per un'analisi della sovrapposizione delle figure di pater e di patrono che Cicerone sceglie come strategia difensiva nei confronti di Celio: se Cicerone tecnicamente era responsabile solo della formazione forense del giovane, qui egli pare sostituirsi al padre naturale di Celio come se avesse il compito della sua educazione in toto.
} 
Si conceda un po' di divertimento all'età, sia la giovinezza più libera; non sia negato tutto ai piaceri, e non sempre abbia la meglio la lucida e severa ragione: di quando in quando la vinca il piacere e il desiderio, purché si abbia anche in questo moderazione e misura. La gioventù sia parca del proprio pudore, non spogli quello degli altri, non dilapidi il patrimonio, né si lasci rovinare dai debiti, non faccia irruzione nelle case e nelle famiglie altrui, non rechi disonore ai casti, rovina agli integri, infamia agli onesti, non minacci nessuno con la violenza, né tenda insidie, stia lontana da ogni delitto. E finalmente, dopo che si sia abbandonata ai piaceri, dopo che sia stato concesso un po' di tempo agli svaghi dell'età e a questi folleggiamenti della fatua giovinezza, ritorni a tempo debito alla cura della casa, del foro e dello stato, così che dimostri di aver scacciato per sazietà e disprezzato per esperienza quelle attività che prima non aveva valutato con la ragione.

Leigh ha mostrato come in quel aliquid temporis sia racchiuso il nocciolo della teoria di Cicerone nei confronti delle intemperanze della gioventù $^{36}$ : che sono da considerare con indulgenza solo nella convinzione che si tratti di follie di breve durata, destinate ad esaurirsi con il passare del tempo. Va da sé il parallelismo con il Micione terenziano, che è comprensivo, sì, ma, come si è visto, solo perché fiducioso in una naturale evoluzione del figlio verso un comportamento più responsabile, una volta venuti meno $\mathrm{i}$ bollori dell'età ${ }^{37}$. Cicerone non solo segue questo modello, ma, se vogliamo, lo mitiga ulteriormente, dal momento che la lista delle azioni proibite che un giovane non deve commettere ricorda proprio i misfatti di Eschi$\mathrm{ne}^{38}$. Sembra quasi, insomma, che la posizione di Cicerone, più che quella di Micione, ricalchi quella del Demea di fine commedia, presentandosi come un'equilibrata mistura di liberalità ed intransigenza.

Risulta chiaro, adesso, spiegare le ragioni di un'assenza importante nell'orazione che pure si pone come la più tollerante e 'open-minded' pronunciata da Cicerone nei confronti dei giovani: l'assenza di lepidus. Ponendosi in perfetta continuità con Terenzio, Cicerone bandisce il senex lepidus dalla sua 'commedia' e conseguentemente anche dal suo modello ideale di rapporto intergenerazionale: condiscendenza ed affabilità non significano complicità e connivenza, né tantomeno attiva riproposizione di comportamenti sbandati che possono essere tollerati solo pro tempore, e non come

36 Vd. Leigh 2004b, pp. 314-324.

37 Vd. Leigh 2004b, p. 323, che nota l'analogia lessicale tra l'asserzione di Micione già analizzata in precedenza (v. 152, sperabam iam defervisse adulescentiam) e Cic. Cael. 77: iam ista deferverint, iam aetas omnia, iam res, iam dies mitigarit ("presto queste cose saranno sbollite, presto l'età, le occupazioni, il tempo mitigheranno ogni cosa").

Come nota ancora Leigh 2004b, p. 322. 
ideale di vita. Così, se Terenzio fa uso della denominazione di lepidus nella caricatura grottesca di Micione recitata da Demea, Cicerone preferisce passare il termine sotto silenzio. Non che non lo conosca, anzi: lo evita appositamente, perché si trattava di un vocabolo molto alla moda, che lui stesso, e proprio per apostrofare i 'nemici' con disprezzo, non manca di usare in altri contesti decisamente meno inclini al dialogo con la gioventù ${ }^{39}$ :

\section{Cic. Catil. 2, 23}

Postremum autem genus est non solum numero verum etiam genere ipso atque vita quod proprium Catilinae est, de eius dilectu, immo vero de complexu eius ac sinu; quos pexo capillo, nitidos, aut imberbis aut bene barbatos videtis, manicatis et talaribus tunicis, velis amictos, non togis; quorum omnis industria vitae et vigilandi labor in antelucanis cenis expromitur. In his gregibus omnes aleatores, omnes adulteri, omnes impuri impudicique versantur. Hi pueri tam lepidi ac delicati non solum amare et amari neque saltare et cantare sed etiam sicas vibrare et spargere venena didicerunt.

Per ultima viene - e non solo in ordine di enumerazione, ma anche per lo stile di vita condotto - la categoria che è propria di Catilina, la sua prediletta, proprio quella che gli sta più a cuore; li vedete ben pettinati, eleganti, o senza barba o con la barba ben curata, con tuniche dalle lunghe maniche, che scendono fino ai piedi, avvolti non da toghe, ma da vestimenti così ampi da sembrare vele; tutta l'attività della loro vita e la fatica della veglia è spesa in banchetti antelucani. In queste greggi si trovano tutti i giocatori d'azzardo, tutti gli adulteri, tutti i dissoluti e gli svergognati. Questi fanciulli tanto graziosi e delicati non hanno imparato solo ad amare e ad essere amati e a danzare e cantare ma anche a vibrare pugnali e spargere veleni.

Il passo è molto conosciuto e prende di mira i difetti della gioventù contemporanea, nelle cui fila è stato facile per Catilina trovare accoliti: ragazzini di per sé indifferenti se non sprezzanti delle questioni di stato, dediti solo a svaghi e dissolutezze, che l'inesperienza politica aveva reso le prede più adatte ad essere irretite dalle lusinghe della rivoluzione. Un conto è perdere la testa per una donna e magari sperperare del denaro per

39 Si rimanda qui all'importante contributo di Krostenko 2004, che mostra come lepos e derivati, insieme ad altri vocaboli propri dell'ideologia catulliana, fossero di per sé vocaboli dalla connotazione ambigua, che Catullo adotta solo in senso positivo, ma che in Cicerone mostrano tutta questa loro ambivalenza di significati: il lepos, così, è una caratteristica del tutto lodevole in un oratore (vd. Cic. de orat. 1, 17), ma diventa riprovevole laddove indichi il comportamento sociale di chi antepone l'otium alla cura dello stato. 
festini e regali; un altro, invece, è dimenticare totalmente il proprio dovere di cittadini romani e dedicarsi esclusivamente ad uno stile di vita molle ed effeminato - questa è l'accezione del tutto negativa con cui Cicerone usa lepidi - che si perde dietro ad amorazzi e convivi. Se si ritorna alle doti di Periplectomeno come amante (v. 656, Venerem, amorem amoenitatemque accubans exerceo, "a tavola mi dedico a Venere, all'amore e al piacere") e ballerino (v. 668, tum ad saltandum non cinaedus malacus aequest atque ego, "nel danzare non c'è ballerino flessuoso che mi sia alla pari"), non vi è dubbio che Cicerone lo avrebbe condannato in toto. Qualcun altro, invece, lo avrebbe esaltato e decantato alla maniera usata da Palestrione nel Miles: mi riferisco, naturalmente, a Catullo, che è molto esperto nell'individuare chi sia degno di essere definito leporum disertus / puer ac facetiarum (Catull. 12, 8-9), e che sicuramente faceva parte di quella jeunesse dorée criticata da Cicerone.

Molti studi sono stati dedicati al lepos di Catullo e alle sue molteplici valenze: lepidus, così, è usato sia in riferimento alla grazia e all'eleganza dei versi del poeta e dei suoi amici, sia alla bellezza di Lesbia che alla sagacia della donna amata o di altri personaggi; non mancano, infine, usi più grossolani e 'comici' del termine, in cui esso sembra associato per lo più ad un divertimento di tipo erotico-sessuale ${ }^{40}$. Ora, però, sulla scia di quanto argomentato fin qui, pare lecito affermare che il lepidum catulliano possa essere letto anche come espressione di 'lepiditas', ovvero come la parola chiave (o una delle parole chiave) a cui Catullo affida il manifesto non solo di una nuova poesia, ma di un nuovo stile di vita paragonabile a quello decantato da Periplectomeno: tutto amore, poesia, vino, amicizia. Tutto otium e niente negotium.

Da questo punto di vista anche il verso d'apertura del liber catulliano può essere letto in modo un po' diverso dal solito: cui dono lepidum novum libellum? Il poeta cerca un destinatario ${ }^{41}$ che sia adatto a ricevere un libro non solo elegante e curato, ma anche moderno, 'al passo coi tempi', e lo trova e lo riconosce nell'amico e storico Cornelio Nepote, un intellettuale aperto alle idee che venivano dalla Grecia, capace di comprendere ed apprezzare sia la novità formale, sia quella contenutistica delle nugae di Catullo. Come si è constatato nel Miles gloriosus, anche qui, dunque, ci troviamo di fronte ad un'opera di giovani per giovani, di amici per amici, che esclude programmaticamente dal proprio orizzonte di riferimento vincoli familiari, sociali, istituzionali, e che si chiude nell'orizzonte di un ludus perenne; unici maestri accettati sono quelli, come Cornelio, che si mos-

40 Per le varie categorie di lepos in Catullo e i relativi luoghi testuali, vd. Seager 1974, Krostenko 2004, Bellandi 2007, pp. 33-38 e 51-61.

41 Sull'importanza del carme 1, 1 di Catullo come testo che problematizza e tematizza l'atto della dedica vd. Schwindt [in corso di stampa]. 
trano compiacenti nei confronti di questa rivoluzione dei gusti. Per questo lepidum, il primo aggettivo che compare in riferimento al libellum, non si limita a sottolinearne la grazia e la cura formale, ma ne mostra la modernità - per questo è accompagnato da novum - e dichiara una sorta di sfida sociale: la 'lepiditas' di Periplectomeno, che era solo un esperimento bizzarro della fantasia plautina, diviene in Catullo una presa di posizione politica ${ }^{42}$, definendo l'ideale di vita di chi si pone di proposito contro il mondo dei senes austeri e delle loro tradizioni e decide di starne fuori per sempre.

Persino i giovani delle commedie di Plauto e di Terenzio accettano di essere ribelli 'a tempo determinato'. Sanno che il gioco delle parti richiede loro una dose di ribellione che sarebbe poi stata messa da parte con l'assunzione di quegli obblighi sociali che non vengono rifiutati per sempre ( $\mathrm{si}$ ricordino le parole di Pleusicle circa la famiglia e i figli). Il proposito dei neoteroi, invece, o quantomeno di Catullo, è di vivere nella commedia per tutta la vita, di protrarre la giovinezza e le sue intemperanze indefinitamente:

Catull. 5

Vivamus, mea Lesbia, atque amemus, rumoresque senum severiorum omnes unius aestimemus assis.

Soles occidere et redire possunt: nobis, cum semel occidit brevis lux, nox est perpetua una dormienda. Da mi basia mille, deinde centum, dein mille altera, dein secunda centum, deinde usque altera mille, deinde centum.

Dein, cum milia multa fecerimus, conturbabimus illa, ne sciamus, aut nequis malus invidere possit, cum tantum sciat esse basiorum.

Viviamo, Lesbia mia, e amiamoci, e liquidiamo tutte le chiacchiere da quattro soldi dei vecchi severi. Il sole può sorgere e tramontare: a noi mortali, una volta che sia venuta meno la breve luce della vita, tocca addormentarci per un'unica notte senza fine. Dammi mille baci, e poi cento, e poi altri mille, e poi duecento, e poi di nuovo mille, e poi cento. Poi, quando avremo raggiunto molte migliaia di baci, mescoliamole insieme,

\footnotetext{
42 In accordo con quanto sostenuto da Krostenko 2004b, che ha coniato l'etichetta di "political aestheticism" per indicare la risemantizzazione catulliana di termini quali lepidus, venustus, bellus, facetus, che, originariamente indicanti qualità non essenziali del discorso oratorio, diventano in Catullo il centro ideologico intorno a cui si definisce l'identità politica del ristretto gruppo dei suoi sodales.
} 
per non saperne più il numero, e affinché nessun spirito malevolo possa gettarci il malocchio, se viene a sapere una quantità così grande di baci.

Nel famoso carme dei baci pare di riconoscere il manifesto della ' $l e$ piditas'. Pur mancando esplicitamente la parola che ne segnerebbe la presenza 'ufficiale' nel testo, il poeta vagheggia un amore che, sfidando la certezza della morte, permetta al poeta e alla donna amata di godere delle brevi gioie dell'esistenza. Nell'innumerabilità dei baci si esprime il desiderio di allontanare il pensiero della fine: che la commedia non termini mai, questo è il sogno di Catullo, che la trasgressione sia la norma, che i vecchi severi e gli invidiosi rimangano fuori dal tempo tutto particolare concesso ai due giovani amanti e a loro soltanto.

E proprio questa secessione dei giovani dalla realtà che teme Cicerone nella Pro Caelio, quando tenta di avvicinarsi alle nuove generazioni con la maschera non del senex severus, i cui rumores uno come Catullo disprezzerebbe, ma con quella gentile del senex lenis. Micione sì, Periplectomeno, però, no, a nessun costo: il senex lepidus plautino si rivela, ancora una volta, una figura impossibile, allontanata da Terenzio e Cicerone, dimenticata dai neoteroi, che si chiudono nel mondo privato dei sodales. Con loro la 'lepiditas' di Periplectomeno continua a vivere, ma passa di diritto ad essere proprietà dei giovani. È così che scompare il senex lepidus - quest'entità ossimorica per definizione, che solo la fantasia estrosa di Plauto aveva avuto il coraggio di ritenere pensabile e mettere in scena - e al suo posto subentrano i pueri lepidi.

\section{Il naufragio della 'lepiditas'}

Se in età tardo-repubblicana la fattibilità di un progetto educativo fondato sulla 'lepiditas' e incarnato dal senex lepidus fallisce del tutto, rimane, però, di tale concezione, l'idea che il lepidum regoli o comunque serva a definire i rapporti tra vecchi e giovani: lepidus è chi fa propria la causa di un modo di vivere anticonformista e liberale quale è quello degli adulescentes. Se nella generazione di Catullo e Cicerone appare evidente che questo tipo di comportamento nelle persone anziane non è né possibile né desiderabile, lepidi sono soltanto i giovani, e la loro 'lepiditas' diventa il vessillo da issare contro, e non più in collaborazione, con i senes stessi.

In conclusione, dunque, se si può dire che l'esperimento tentato da Plauto con Periplectomeno rimanga un caso più unico che raro, destinato a non avere seguaci, è anche vero, però, che il suo esempio propone una duplice concezione del lepidum, che, come si è accennato in apertura, ha avuto dei lunghi riflessi nella società repubblicana romana: da una parte esso incarna il carattere giocoso e carnevalesco del teatro e della lettera- 
tura in generale, l'esperimento audace e divertente che rompe con la normalità e scatena il riso; dall'altra, e come conseguenza di ciò, esso diventa uno stile di vita potenzialmente pericoloso. Ebbene, questa seconda caratterizzazione, che, per così dire, propone un modello sociale e si presta ad essere fatta propria dai giovani, viene meno in età imperiale e il vocabolo conosce un rapido declino.

Lepidus, ad esempio, scompare del tutto negli autori elegiaci, che pure una sola generazione separa da Catullo, e che si muovevano in continuità con le sue idee, portandone a perfetto compimento la scelta dell'amore come unico scopo e centro dell'esistenza. Assente anche in altri autori 'ribelli' come Petronio, Giovenale e Persio, si ritrova in Marziale, ma sembra marcare, ormai, solo un genere minore, la facezia che fa ridere, la notizia pruriginosa, la storiella adulterina ${ }^{43}$. In Apuleio, invece, lepidus incontra addirittura il maggior numero di attestazioni dopo Plauto ${ }^{44}$, tanto che sorprende che non sia stato inserito tra i numerosi elementi del già indagato 'plautino in Apuleio'45. I contesti in cui il termine appare, tuttavia, sono totalmente privi della seconda accezione che è stata qui individuata, come, del resto, lo è l'intera vicenda, che, pur presentando le disavventure di un giovane indisciplinato, non tematizza affatto il problema dello scontro generazionale. Il lepidum, nella storia di Lucio, appare più che altro come l'esca sagace e piccante che introduce un nuovo racconto. Con tale esca il narratore dapprima scatena la curiosità del lettore, invitandolo a iniziare la lettura del libro, e poi, nel corso del romanzo, di Lucio stesso, che anela di ascoltare ogni storia lepida che gli venga fatta fiutare:

Apul. Met. 1, 1

At ego tibi sermone isto Milesio varias fabulas conseram auresque tuas benivolas lepido susurro permulceam.

E ora intreccerò per te in questo racconto del genere milesio varie novelle, e accarezzerò le tue benevole orecchie con il mio divertente chiacchiericcio.

Apul. Met. 1, 2

Ac dum ausculto quid sermonis agitarent, alter exerto cachinno:

43 Qualche esempio: Mart. 8, 3, 19 (at tu Romano lepidos sale tinge libellos, "ma tu spargi di sale romano i libretti piccanti"), Mart. 11, 20, 9-10 (absolvis lepidos nimirum, Auguste, libellos, / qui scis Romana simplicitate loqui, "tu certo, Augusto, perdoni i miei libretti licenziosi, tu che conosci il modo di parlar schietto dei Romani").

44 Vd. Monteil 1964, p. 136, che ritiene che la ragione di ciò sia l'appartenenza del termine ad un lessico di tipo familiare, che Apuleio recupera nel genere minore della favola milesia.

45 Vd. Pasetti 2007, che indaga la profonda influenza di Plauto sul lessico apuleiano. 
"Parce" inquit "in verba ista haec tam absurda tamque immania mentiendo". Isto accepto sititor alioquin novitatis: "Immo vero" inquam "impertite sermone non quidem curiosum sed qui velim scire vel cuncta vel certe plurima; simul iugi quod insurgimus aspritudinem fabularum lepida iucunditas levigabit".

Mentre tento di capire che genere di discorso stavano facendo, uno dei due scoppia a ridere e dice: "Ma smettila di inventare racconti così smisuratamente assurdi!". Udito ciò, io, che, tutto al contrario, ho sempre sete di novità, intervengo: "vi prego, fate partecipe dei vostri discorsi anche me, che, per quanto non sia un curiosone, vorrei sapere, se non proprio tutto, certamente il più possibile. E intanto la piacevole allegria dei racconti renderà più dolce l'asprezza del pendio su cui ci stiamo inerpicando".

\section{Apul. Met. 2, 20}

"Immo mi Thelyphron", Byrrhena inquit et "subsiste paulisper et more tuae urbanitatis fabulam illam tuam remetire, ut et filius meus iste Lucius lepidi sermonis tui perfruatur comitate".

"Ma no, caro Telifrone", disse Birrena, "trattieniti ancora un po', e, con il garbo che ti contraddistingue, raccontaci ancora una volta quella tua famosa storiella, perché anche questo mio figliolo, Lucio, possa godere della piacevolezza del tuo affascinante racconto".

Già da questi pochi esempi pare evidente come lepidus non abbia più nulla del modello di vita, criticato o meno, presentato in Plauto e in Terenzio, né dei riflessi che tale modello poteva esercitare nei rapporti tra giovani e vecchi, così acutamente avvertiti ancora nel primo secolo a. C.; al contrario, esso si riduce ad una sorta di marcatore del genere comico, il segnale che annuncia al pubblico la storiella divertente e disimpegnata. Lo spirito anticonformista e modernizzante con cui Catullo intendeva lepidus, nonché l'estremo riguardo con cui Cicerone lo evitava nella Pro Caelio, sono semplicemente decaduti con il decadere dello scontro generazionale sul finire della repubblica. Non c'è più un Cicerone a cui interessi arruolare giovani nelle file dei boni cives, non ci sono più le condizioni storiche per cui ignorare gli affari di stato e ripiegarsi sul privato possa essere visto come una pericolosa forma di ribellione e non come una semplice necessità.

Del senex lepidus impersonato da Periplectomeno era destinato a sopravvivere solo l'aspetto più volgarmente comico che lo avvicinava ai senes amatores, e non l'utopia di una vita eternamente giovane ed eternamente folle. Ugualmente, dell'aggettivo lepidus si conservò la patina provocante 
e ammiccante, ma nulla della sostanza che lo rendeva tale. Prova di questo indebolimento semantico si riscontra anche nella fortuna del termine nell'italiano letterario, dove "lepido" non significa certo "liberale", "al passo coi tempi", "moderno", ma soltanto "divertente", "faceto", "grazioso", in contesti che variano, normalmente, dal sentimentale al licenzioso:

Lorenzo Da Ponte, Così fan tutte, atto secondo, scena seconda, duetto n. 20 DORABELLA. Prenderò quel brunettino, / che più lepido mi par. FIORDILIGI. Ed intanto io col biondino / vo' un po' ridere e burlar. DORABELLA. Scherzosetta, ai dolci detti / io di quel risponderò. FIORDILIGI. Sospirando, i sospiretti / io dell'altro imiterò. DORABELLA. Mi dirà: / ben mio, mi moro! FIORDILIGI. Mi dirà: / mio bel tesoro! FIORDILIGI E DORABELLA. Ed intanto che diletto, / che spassetto / io proverò!

Triste declino di una parola che aveva rappresentato le speranze dei giovani e i timori dei vecchi.

\section{Bibliografia}

BarCHIESI, M. (1957) "Problematica e poesia in Plauto", Maia 9, pp. 191-200. BetTini, M. (1981) "Un'utopia per burla", introduzione a M. Bettini (a cura di), Plauto, Mostellaria, Persa, Milano, pp. 9-23 (ora in Bettini 1991, pp. 77-96).

BettinI, M. (1982) "Verso un'antropologia dell'intreccio. Le strutture semplici della trama nelle commedie di Plauto", Materiali e discussioni per l'analisi dei testi classici 7, pp. 39-101 (ora in Bettini 1991, pp. 11-76). BettinI, M. (1991) Verso un'antropologia dell'intreccio e altri studi su Plauto, Urbino.

Belland, F (2007) Lepos e pathos. Studi su Catullo, Bologna.

Bonnefond, M. A. (1983) "Senato e conflitti di generazioni nella Roma repubblicana", in E. Pellizer, N. Zorzetti (a cura di), La paura dei padri nella società antica e medioevale, Roma-Bari, pp. 67-98.

Citroni Marchetti, S. (2004) "I precetti paterni e le lezioni dei filosofi: Demea, il padre di Orazio ed altri padri e figli", Materiali e discussioni per l'analisi dei testi classici, pp. 9-63.

Duckworth, G. E. (1952) The Nature of Roman comedy: a study in popular entertainment, Princeton.

Eyben, E. (1991) "Fathers and sons", in B. Rawson (ed. by) Marriage, divorce, and children in ancient Rome, Oxford, pp. 114-143. 
Fantham, E. (1971) "Heautontimorumenos and Adelphoe: a study on fatherhood in Terence and Menander", in Latomus 30, pp. 970-998.

FRÄNKEL, E. (1960) Elementi plautini in Plauto, Firenze. Traduzione italiana di Plautinisches im Plautus, Berlin [1922].

Geffrcken, K. A. (1973) "Comedy in the "Pro Caelio", Mnemosyne Supplement 30, Leiden.

Klowski, J. (2000) Terenz'Adelphen und die modernen Erziehungsstile, in Gymnasium 107, pp. 109-127.

Krostenko 2004 = B. A. Krostenko Catullus and Elite Republican Social Discourse, in M. B. Skinner (edited by), A Companion to Catullus, Oxford.

LABATE $2012=$ M. LABATE Vicende della probitas: immaginario teatrale $e$ valori etici, in G. Petrone, M. M. Bianco (a cura di), Immaginari comici, pp. 23-53.

LEFÈVRE 1984 = E. LefÈVRe Die Umformung des Alazon zu der DoppelKömodie des Miles Gloriosus, in Hermes 112, pp. 30-52.

LEIGH 2004A = M. LEIGH Comedy and the rise of Rome, Oxford.

Leigh 2004B $=$ M. Leigh The Pro Caelio and Comedy, in Classical Philology 99, pp. 300-335.

LENTANO 1996 = M. LENTANO Le relazioni difficili: parentela e matrimonio nella commedia latina, Napoli.

Leo 1896 = F. Leo Plauti Comoediae, v. II, Berolini.

LEO 1912 = F. LEO Plautinische Forschungen. Zur Kritik und Geschichte der Kömodie, Berlin.

LieBerg 1988 = G. LieBerg Das pädagogisch-dramatische Problem der Adelphen des Terenz, in Grazer Beiträge. Zeitschrift für die Klassische Altertumswissenschaft 15, pp. 73-84.

LINDSAY 1905 = W. M. LINDSAY T. Macci Plauti Comodiae, v. II, Oxonii.

Maurice $2007=$ L. Maurice Structure and Stagecraft in Plautus' Miles Gloriosus, in Mnemosyne 60, pp. 407-426.

MAY 1995 = J. M. MAY Patron and Client, Father and Son in Cicero's "Pro Caelio", in The Classical Journal 90, pp. 433-441.

MINARINI 1995 = A. MINARINI La palliata, in U. Mattioli (a cura di), Senectus: la vecchiaia nel mondo classico, 2, Bologna, pp. 1-30.

MINOIS 1988 = G. MINOIs Storia della vecchiaia dall'antichità al rinascimento,

Bari. Traduzione italiana di Histoire de la vieillesse en Occident de l'Antiquité à la Renaissance, Paris 1987.

Monteil 1964 = P. Monteil Beau et laid en latin. Étude de vocabulaire, Paris. Morenilla 1993 = C. MorenILla Periplectómeno: la aristeia de una vieja figura cómica, in Emerita 61, pp. 61-94.

NARDUCCI $1989=\mathrm{E}$. NARDUCCI Cicerone e la gioventù romana, introduzione a Cicerone, Difesa di Marco Celio, Milano, pp. 5-55. 
NARDUCCI $2009=$ E. NARDUCCI Le trasgressioni della gioventù. Il processo di Marco Celio, in E. Narducci, Cicerone: la parola e la politica, Roma, pp. 257-276.

Neraudau 1979 = J. P. Neraudau La jeunesse dans la littérature et les institutions de la Rome républicaine, Paris.

Paratore 1959 = E. Paratore, introduzione a Plauto, Miles Gloriosus, Firenze. PAsetTI $2007=$ L. PAsettI Plauto in Apuleio, Bologna.

Petrone 1977 = G. Petrone Morale e antimorale nelle commedie di Plauto, Palermo.

Raccanelli 1998 = R. Raccanelli L'amicitia nelle commedie di Plauto: un'indagine antropologica, Bari.

Ryder 1984 = K. C. Ryder The "Senex Amator" in Plautus, in Greece E Rome 31, pp. 181-189.

SAYLOR $1977=$ C. SAYLOR Periplectomenus and the organization of the Miles gloriosus, in Eranos 75, pp. 1-13.

SchaAF 1977 = L. SchaAf Der Miles Gloriosus des Plautus und sein griechisches Original: ein Beitrag zur Kontaminationsfrage, München.

SCHWINDT [IN CORSO DI STAMPA] = J. P. SCHWINDT Die Dinge der Dichtung. Zur Anatomie der Schriftlichkeit in Catulls libellus (relazione tenuta nel 2013 al simposio "Wissen in materialen Textkulturen", organizzato dal Deutscher Literaturarchiv Marbach e dal gruppo di ricerca "Materiale Textkulturen").

Seager 1974 = R. Seager, "Venustus, Lepidus, Bellus, Salsus": Notes on the Language of Catullus, in Latomus 33, pp. 891-894.

TRÄNKLE $1972=\mathrm{H}$. TRÄNKLE Micio und Demea in terenzischen Adelphen, in Museum Helveticum 29, pp. 241-255.

ZwIERLEIN 1991 = O. ZwIERLEIN Zur Kritik und Exegese des Plautus, v. II, Miles gloriosus, Stuttgart. 